\title{
Impact of Neoadjuvant Chemotherapy on the Administration of Concurrent Chemoradiation for Locally Advanced Nasopharyngeal Carcinoma
}

\author{
Benjamin Maas ${ }^{1}$, Cheryl Ho ${ }^{2}$, Sarah Hamilton ${ }^{1}$, Doug Leedy ${ }^{3}$, Eric Berthelet ${ }^{1}$ \\ 1. Radiation Oncology, British Columbia Cancer, Vancouver Cancer Centre, Vancouver, CAN 2. Medical \\ Oncology, British Columbia Cancer, Vancouver Cancer Centre, Vancouver, CAN 3. Medicine, University of \\ British Columbia, Vancouver, CAN
}

$\square$ Corresponding author: Benjamin Maas, benjamin.maas@bccancer.bc.ca Disclosures can be found in Additional Information at the end of the article

\section{Abstract \\ Objectives}

The standard of care for locally advanced nasopharyngeal carcinoma (NPC) is concurrent cisplatin chemoradiotherapy. Neoadjuvant chemotherapy can be administered to downsize tumors before concurrent treatment to optimize radiation volumes. Our hypothesis was that the use of cisplatin in the neoadjuvant phase could limit the amount of cisplatin that patients could tolerate in the concurrent phase of treatment.

\section{Methods}

This is a retrospective analysis of Canadian NPC patients who received neoadjuvant chemotherapy with the intention to downsize locally advanced tumors prior to concurrent cisplatin plus radiation. Baseline demographic and treatment data were obtained from institutional databases and chart review; all data were analyzed with SPSS (SPSS Inc. Released 2005. SPSS for Windows, Version 14.0. Chicago: SPSS Inc.) Overall survival (OS), diseasespecific survival (DSS), and local/regional relapse-free survival (LRRFS) were analyzed using Kaplan-Meier survival functions. Univariate and multivariate models were used to determine factors associated with the total dose of concurrent chemotherapy.

\section{Results}

Forty-six patients were identified as receiving neoadjuvant chemotherapy before concurrent chemoradiotherapy. In the univariate and multivariate analyses of patients who received

Received 08/18/2017

Review began 09/27/2017 Review ended 07/06/2018 Published 07/12/2018

\section{(C) Copyright 2018}

Maas et al. This is an open access article distributed under the terms of the Creative Commons Attribution License CC-BY 3.0., which permits unrestricted use, distribution, and reproduction in any medium, provided the original author and source are credited. concurrent chemotherapy, receiving over $200 \mathrm{mg} / \mathrm{m}^{2}$ concurrent cisplatin with radiation was associated with a higher neoadjuvant dose of chemotherapy received. The median follow-up time was 2.6 years (range, 0.17 years to 10.6 years). At three years, the OS was $83 \%$, DSS was $86 \%$, and LRRFS was $74 \%$.

\section{Conclusions}

NPC patients have been treated with neoadjuvant chemotherapy at this center with favorable outcomes. Most patients could tolerate concurrent chemotherapy after radiotherapy. Receiving higher doses of concurrent chemotherapy was associated with also having higher doses of neoadjuvant cisplatin. This suggests that neoadjuvant cisplatin is not a limiting factor in the delivery of full-dose concurrent chemotherapy.

How to cite this article

Maas B, Ho C, Hamilton S, et al. (July 12, 2018) Impact of Neoadjuvant Chemotherapy on the Administration of Concurrent Chemoradiation for Locally Advanced Nasopharyngeal Carcinoma. Cureus 10(7): e2971. DOI 10.7759/cureus.2971 
Categories: Otolaryngology, Radiation Oncology, Oncology

Keywords: nasopharyngeal carcinoma, neoadjuvant chemotherapy, concurrent chemotherapy

\section{Introduction}

Radiotherapy is the mainstay of treatment for nasopharyngeal carcinoma (NPC) [1-2]. Doses of $70 \mathrm{~Gy}$ directed to the primary tumor and involved lymph nodes have been recognized as a curative treatment protocol. In the locally advanced setting, concurrent chemoradiotherapy is the standard of care based on Phase III trials conducted in both North America and Asia that demonstrated a significant survival benefit [3-4]. The most common radiosensitizing agent is cisplatin delivered at $40 \mathrm{mg} / \mathrm{m}^{2}$ weekly or $100 \mathrm{mg} / \mathrm{m}^{2}$ every three weeks. The roles of neoadjuvant and adjuvant chemotherapy remain controversial, with conflicting results throughout the literature [1,5-9].

Neoadjuvant chemotherapy has been used for locally advanced presentations of NPC. The proposed benefits of neoadjuvant chemotherapy in this setting are to downsize the tumor prior to radiation and to treat microscopic metastatic disease. In bulky Stage III and Stage IV-A and IV-B tumors, encompassing the gross tumor volume (GTV) with a curative dose of 70 Gy may prove challenging due to the proximity of dose-limiting structures, such as the spinal cord, brain stem, brain, and optic structures. Downsizing from neoadjuvant chemotherapy may help shrink the tumor away from dose-limiting critical structures and, therefore, facilitate radiation delivery and reduce the risk of significant morbidity or even mortality.

There are cumulative toxicities associated with higher total doses of platinum-based chemotherapy, including ototoxicity, nephrotoxicity, and neuropathy. It is unclear if increasing the total chemotherapy dose by adding a neoadjuvant phase limits a patient's ability to complete the curative-intent concurrent treatment phase. Because the efficacy of concurrent chemoradiotherapy for NPC has been well-demonstrated, it is important to assess whether neoadjuvant platinum-based chemotherapy lowers a patient's ability to receive concurrent chemotherapy with radiation.

At the British Columbia Cancer Agency (BCCA), selected patients with bulky NPC primary tumors at diagnosis have been treated with the neoadjuvant chemotherapy phase prior to definitive concurrent chemoradiation. We conducted a retrospective analysis to identify all such patients and report on the clinical outcomes. The primary objectives were to present a Canadian experience with neoadjuvant chemotherapy and to characterize the subsequent ability to complete the intended and prescribed concurrent chemotherapy during radiation. The secondary objectives were to report the disease recurrence and survival outcomes for the patients treated.

\section{Materials And Methods}

A retrospective review was conducted of patients diagnosed with NPC treated at the BCCA from January 1, 2000, to December 1, 2013, who received chemotherapy prior to planned curative intent concurrent chemoradiation treatment. The BCCA has six regional centers and provides comprehensive oncologic care to a population of 4.5 million people, which includes chemotherapeutic drugs and all radiation therapy. This large population and single radiation provider allow for prospective data capture on treatments and outcomes for a population-based assessment of outcomes.

Demographic data obtained from patient medical records included age, sex, ethnicity, date of 
birth, smoking status, and Eastern Cooperative Oncology Group (ECOG) performance status. Disease characteristics obtained included tumor-node-metastasis (TNM) stage, American Joint Committee on Cancer 2009 stage, and International Classification of Diseases diagnosis codes.

Neoadjuvant chemotherapy was defined as any chemotherapy regimen received prior to concurrent chemoradiotherapy. All neoadjuvant protocols were platinum-based. Patients were selected for neoadjuvant chemotherapy if they had bulky T3/T4 disease invading or abutting the brain, optic structures, brain stem, or spinal cord to cytoreduce the bulk of disease prior to the delivery of radiotherapy. All patients were assessed by a medical oncologist for suitability for neoadjuvant chemotherapy and were ECOG 0-2 with adequate hematologic, hepatic, and renal function. Standard concurrent chemotherapy regimens utilized were cisplatin $40 \mathrm{mg} / \mathrm{m}^{2}$ weekly for seven cycles or cisplatin $100 \mathrm{mg} / \mathrm{m}^{2}$ every three weeks for three cycles. Treatment data were compiled, including start date, end date, chemotherapy agents (neoadjuvant and concurrent), the total dose of cisplatin in $\mathrm{mg} / \mathrm{m}^{2}$, and the number of cycles. Radiotherapy is most commonly prescribed as $70 \mathrm{~Gy}$ in 35 daily fractions five days per week, with some physician variation in dose and fractionation.

A cutoff of $\leqslant 200 \mathrm{mg} / \mathrm{m}^{2}$ or $>200 \mathrm{mg} / \mathrm{m}^{2}$ of cisplatin received during the concurrent phase was used to stratify the cohort. This was selected as a marker for completing at least two thriceweekly $100 \mathrm{mg} / \mathrm{m}^{2}$ cycles or five weekly $40 \mathrm{mg} / \mathrm{m}^{2}$ cycles of once weekly concurrent cisplatin. Univariate and multivariate models were used to determine factors associated with receiving < $200 \mathrm{mg} / \mathrm{m}^{2}$ of concurrent chemotherapy.

Secondary endpoints included overall survival (OS), disease-specific survival (DSS), and local/regional relapse-free survival (LRRFS). OS was defined as the duration from diagnosis to the date of death from any cause or censored at last date of follow-up. DSS was defined as the time from diagnosis to death from NPC. Local recurrence was defined as any recurrence in the nasopharynx after a treatment response as identified by clinical exam, imaging, or biopsy. Regional recurrence was defined as any recurrence in regional nodes. LRRFS was defined as time to a local or regional recurrence.

Categorical variables were compared using Fisher's exact test, and continuous variables were analyzed using the Mann-Whitney test. Survival was analyzed with Kaplan-Meier survival functions. The three-year survival endpoints were extracted. Patients with longer than three years of follow-up were censored at three years for this subanalysis. Statistical analysis was conducted using SPSS (SPSS Inc. Released 2005. SPSS for Windows, Version 14.0. Chicago: SPSS Inc.).

The study was reviewed and approved by the BCCA Research Ethics Board.

\section{Results}

From January 2000 to December 2013, 428 cases of NPC were identified at the BCCA. Of these, 46 patients fit the inclusion criteria of receiving neoadjuvant chemotherapy prior to planned concurrent chemoradiotherapy. The baseline characteristics of the 46 patients, including demographic and tumor staging information, are found in Table 1 . The majority of patients were male, of Asian ethnicity, and had T3 or T4 tumors with involved nodes. 


\section{Cureus}

Range: $18-75$ years

$\begin{array}{lll}\text { Gender } & \text { Actual } & \text { Percent } \\ \text { Female } & 9 & 20 \% \\ \text { Male } & 37 & 80 \%\end{array}$

Ethnicity

Caucasian

ECOG Performance Status

\section{Tumor Stage}

$7 \%$

Nodal Stage

$7 \%$

\section{Metastases}

Stage

III

Smoking Status

Current 


\section{Cureus}

Former

Never
12

22

4
$9 \%$

\section{TABLE 1: Patient and tumor characteristics}

Abbreviations: ECOG, Eastern Cooperative Oncology Group.

Treatment details are outlined in Table 2. The most common neoadjuvant chemotherapy combination was cisplatin and gemcitabine. The median dose of neoadjuvant cisplatin was 160 $\mathrm{mg} / \mathrm{m}^{2}$ (range, $24 \mathrm{mg} / \mathrm{m}^{2}$ to $325 \mathrm{mg} / \mathrm{m}_{2}$ ). Forty-three patients were scheduled to receive cisplatin $40 \mathrm{mg} / \mathrm{m}^{2}$ weekly for seven cycles, and three patients were scheduled to receive cisplatin $100 \mathrm{mg} / \mathrm{m}^{2}$ every three weeks for three cycles. Of the 46 patients, $52 \%$ received all planned concurrent cisplatin. The median dose of concurrent cisplatin was $195 \mathrm{mg} / \mathrm{m}^{2}$ (range, $67 \mathrm{mg} / \mathrm{m}^{2}$ to $397 \mathrm{mg} / \mathrm{m}^{2}$ ). Twenty-one of 46 patients received $\leqslant 200 \mathrm{mg} / \mathrm{m}^{2}$ concurrent cisplatin, including four patients who did not receive any of the concurrent phase planned cisplatin. Some patients who received less than $70 \mathrm{~Gy}$ were due to the proximity of the tumor to organs of interest (OARs); no patient stopped radiation treatment early due to toxicity. One patient declined radiation due to fear of potential side effects. 


\section{Cureus}

\section{Neoadjuvant Chemotherapy Agents}

Gemcitabine \& Cisplatin

Etoposide \& Cisplatin

Fluorouracil \& Cisplatin

Concurrent Chemotherapy (Planned Treatment)

Cisplatin $40 \mathrm{mg} / \mathrm{m}^{2}$ weekly $\times$

Number of patients who received all planned Cisplatin

Cisplatin Dose Stratified

$$
\begin{aligned}
& \leq 200 \mathrm{mg} / \mathrm{m}^{2} \text { (Range: } 0-200 \mathrm{mg} / \mathrm{m}^{2} \text { ) } \\
& >200 \mathrm{mg} / \mathrm{m}^{2} \text { (Range: } 201-320 \mathrm{mg} / \mathrm{m}^{2} \text { ) } \\
& \text { Radiation Dose and Fractionation }
\end{aligned}
$$

70 Gy / 35\#

70 Gy / 36\#

70 Gy / 33\#

66 Gy / 33\#

60 Gy / 30\#

60 Gy / 25\#

56 Gy / 28\#

Declined RT

\section{Number of Patients}

40

3

3

$6.5 \%$

$94 \%$

$52 \%$

TABLE 2: Neoadjuvant chemotherapy regimen, dose of concurrent chemotherapy, and radiation dose fractionation schedule

Abbreviations: $\mathrm{RT}$, radiotherapy.

Of the 46 patients who received neoadjuvant chemotherapy, 45 went on to receive radiation therapy. One patient refused any radiation treatment after the neoadjuvant treatment phase. All were given radical radiotherapy courses, but there was some variability in the dose and fractionation based on provider choice and patient-specific factors. Radiation doses ranged from $56 \mathrm{~Gy}$ to $70 \mathrm{~Gy}$ in 25 to 36 daily fractions (Table 2). The most common dose fractionation was $70 \mathrm{~Gy}$ in 35 fractions for 33 patients (72\%).

Potential factors associated with completing $>200 \mathrm{mg} / \mathrm{m}^{2}$ concurrent phase cisplatin were 


\section{Cureus}

analyzed in univariate and multivariate models presented in Table 3. Receiving a higher dose of neoadjuvant chemotherapy was significantly associated with completing $>200 \mathrm{mg} / \mathrm{m}^{2}$ concurrent cisplatin in the univariate analysis. Males also trended towards higher concurrent chemotherapy strata with a p-value of 0.059 . Stage, age, ECOG performance status, and ethnicity were not predictive of completing concurrent chemotherapy.

\begin{tabular}{|c|c|c|c|c|c|}
\hline & \multicolumn{3}{|l|}{ Univariate } & \multicolumn{2}{|l|}{ Multivariate } \\
\hline & $\leq 200 \mathrm{mg} / \mathrm{m}^{2}$ & $>200 \mathrm{mg} / \mathrm{m}^{2}$ & $p$-value & Odds Ratio & $\mathrm{p}$-value \\
\hline \multicolumn{6}{|l|}{ Sex } \\
\hline Female & $6(35 \%)$ & $2(8 \%)$ & 0.045 & 4.9 & 0.027 \\
\hline Male & $11(65 \%)$ & $23(92 \%)$ & & & \\
\hline \multicolumn{6}{|l|}{ Ethnicity } \\
\hline Caucasian & $5(30 \%)$ & $4(16 \%)$ & 0.45 & NA & NA \\
\hline Asian & $12(71 \%)$ & $21(84 \%)$ & & & \\
\hline \multicolumn{6}{|l|}{ Stage } \\
\hline III & $5(29 \%)$ & $7(28 \%)$ & 1 & & \\
\hline IV & $12(71 \%)$ & $18(72 \%)$ & & & \\
\hline \multicolumn{6}{|l|}{ ECOG Performance Status } \\
\hline 0 & $17(100 \%)$ & $21(84 \%)$ & 0.13 & NA & NA \\
\hline 1 or 2 & $0(0 \%)$ & $4(16 \%)$ & & & \\
\hline \multicolumn{6}{|l|}{ Smoking Status } \\
\hline Current/former & $12(50 \%)$ & $9(50 \%)$ & 0.7 & NA & \\
\hline Never & $9(38 \%)$ & $8(44 \%)$ & & & \\
\hline Unknown & $3(13 \%)$ & $1(6 \%)$ & & & \\
\hline Mann-Whitney Test & Mean Rank & Mean Rank & & & \\
\hline Age & 23.8 & 19.9 & 0.32 & NA & NA \\
\hline Neaodjuvant cisplatin (mg/m²) & 15.6 & 25.5 & 0.01 & 7.2 & 0.007 \\
\hline
\end{tabular}

TABLE 3: Univariate and multivariate by dose stratification

Abbreviations: ECOG, Eastern Cooperative Oncology Group; NA, not applicable. 


\section{Cureus}

univariate models. A linear regression analysis of the continuous dose of cisplatin received neoadjuvantly and the dose of cisplatin received concurrently found a positive correlation; patients who were able to receive higher doses of cisplatin in the neoadjuvant setting also received higher doses of cisplatin in the concurrent setting.

Median follow-up was 2.6 years with a range of 0.2 years to 10 years. Seven patients died during follow-up, and the three-year overall survival (OS) was $83 \%$ (Figure 1). There were six deaths due to NPC, and the three-year DSS was 86\% (Figure 2). Nine patients had documented local or regional recurrences following treatment with a three-year LRRFS of 74\% (Figure 3).

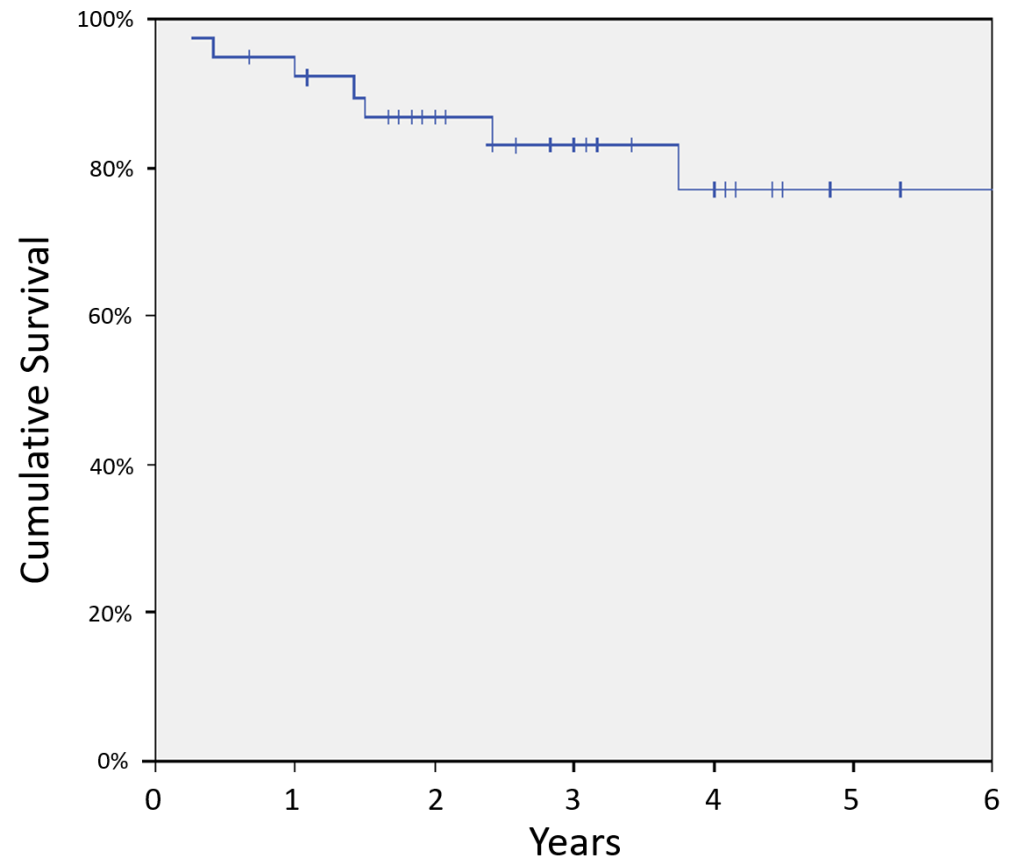

Survival Function

+ Censored

\section{FIGURE 1: Overall survival}

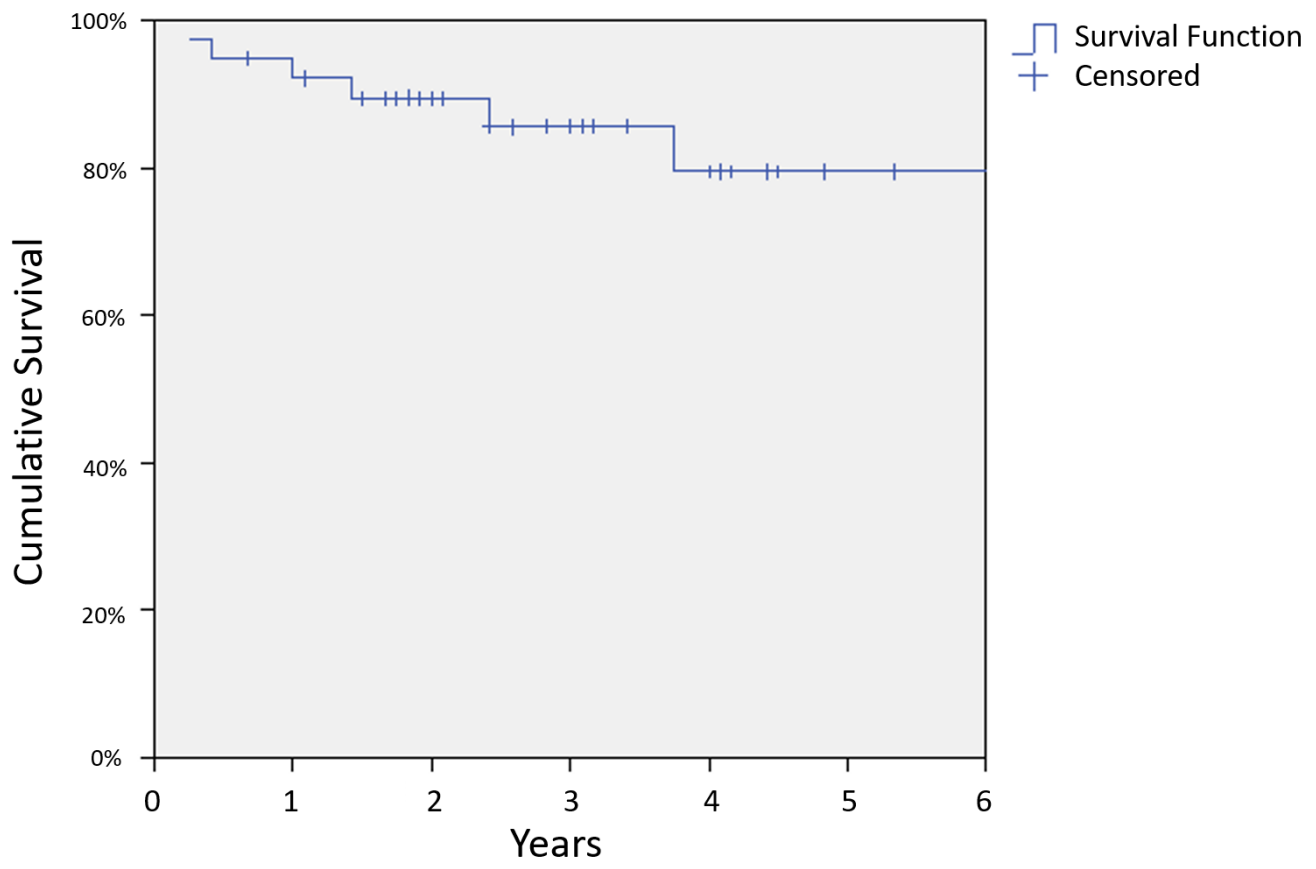




\section{Cureus}

FIGURE 2: Disease-specific survival

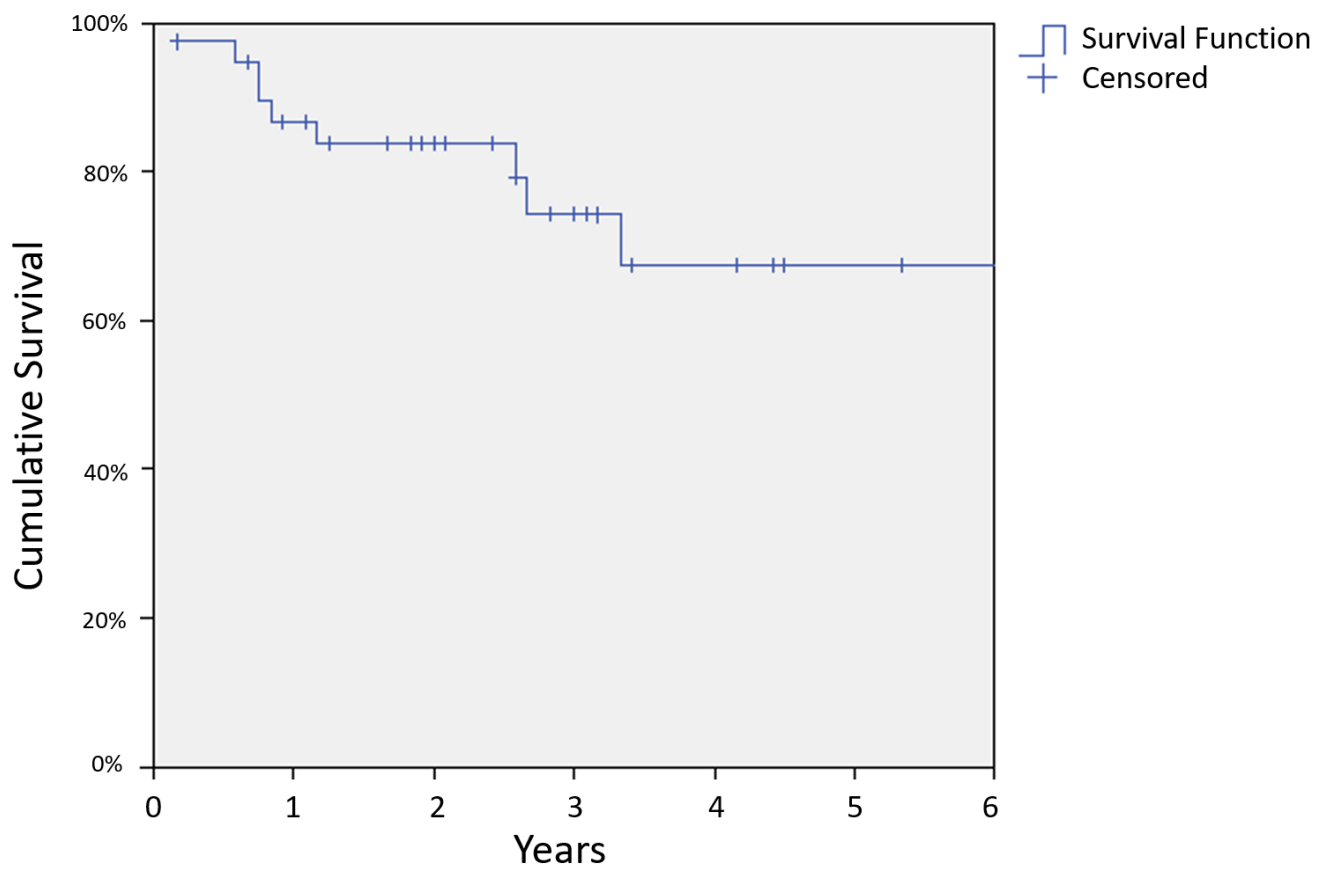

FIGURE 3: Recurrence-free survival

\section{Discussion}

This retrospective study of locally advanced NPC patients evaluated the impact of neoadjuvant cisplatin on concurrent cisplatin chemotherapy dose. Higher doses of neoadjuvant chemotherapy were associated with completing more cycles of concurrent cisplatin during the radiotherapy stage of treatment. This was in opposition to our hypothesis that increasing the overall cisplatin exposure and the associated toxicity would decrease the amount of concurrent cisplatin received during radiation.

Neoadjuvant regimens for NPC are typically platinum-based combinations. The goal of therapy includes reducing the primary tumor and controlling the microscopic metastatic disease.

Multiple Phase II trials have been conducted using cisplatin in combination with anthracyclines, taxanes, and anti-metabolites [7-8,10-11]. The total dose of neoadjuvant cisplatin in these studies ranged from $150 \mathrm{mg} / \mathrm{m}^{2}$ to $240 \mathrm{mg} / \mathrm{m}^{2}$ with a concurrent component total dose of $150 \mathrm{mg} / \mathrm{m}^{2}$ to $300 \mathrm{mg} / \mathrm{m}^{2}$. Despite these cumulatively high doses of cisplatin, compliance with therapy was reported to be high in these patients selected for the clinical trial.

Nephrotoxicity, neuropathy, and ototoxicity have been recognized as treatment-limiting side effects with cisplatin. A cumulative dose of cisplatin of over $400 \mathrm{mg} / \mathrm{m}^{2}$ via bolus administration or concurrent delivery with radiotherapy is known to increase the likelihood of developing these side effects. Established predictive patient factors include increased age and female sex. In our study, the dose of concurrent cisplatin was not negatively impacted using neoadjuvant treatment despite the anticipated higher risk of toxicity. Like other studies, however, male sex appears to be predictive of the ability to receive higher doses of concurrent 
cisplatin.

It is difficult to compare survival outcomes from different studies due to the subgroup of bulky tumors that were selected for this treatment at our center, but the three-year OS rate of $83 \%$ for locally advanced NPC is comparable to several Phase II clinical trials of neoadjuvant chemotherapy prior to radical treatment for NPC [7-8]. Neoadjuvant chemotherapy for NPC has been evaluated prospectively among few patients. Hui et al. reported on a Phase II trial of 65 patients randomized to neoadjuvant treatment for Stage III and IV-B NPC followed by chemoradiation in Hong Kong. The three-year progression-free survival (PFS) reported for neoadjuvant versus control was $88.2 \%$ and $59.5 \%$, and the three-year OS for neoadjuvant versus control was $94.1 \%$ and $67.7 \%$, respectively [8]. Kong et al. in Shanghai reported on three-year PFS and OS for 52 patients with Stage III NPC and 64 patients with non-metastatic Stage IV NPC all treated with neoadjuvant chemotherapy followed by concurrent chemoradiation. They report a three-year PFS at $78.2 \%$ and $85.1 \%$ for Stage III and IV patients, respectively. The three-year OS in this trial was $94.8 \%$ and $90.2 \%$ for Stage III and IV patients, respectively [7]. However, in these two trials, a bulky primary tumor was not part of the inclusion criteria. Nonetheless, these results are comparable to our results. A previous retrospective analysis by Hamilton et al. of NPC patients at the BCCA reported five-year survival outcomes for Stage III and IVB patients at close to our three-year survival results [12].

Limitations of this study design include the fact that it was a retrospective study and all toxicities of neoadjuvant therapy may not have been captured. There could be a selection bias in treatment decisions, both in selecting patients for neoadjuvant therapy who were more fit at diagnosis and patients with a high tumor stage for cytoreduction. The neoadjuvant and concurrent chemotherapy schedules and regimens were not uniform; however, we were able to collect the total cisplatin doses in each phase. The total dose of cisplatin $>200 \mathrm{mg} / \mathrm{m}^{2}$ as a marker for tolerating concurrent cisplatin chemotherapy was chosen based on existing literature and may not be the best marker for clinical effectiveness. Despite these design limitations, this is a fairly large cohort of locally advanced NPC patients. So far, studies of neoadjuvant chemotherapy before concurrent chemoradiation have been limited, particularly in the North American setting. There are currently ongoing Phase III randomized controlled trials (Hong Kong, Singapore, and France) addressing neoadjuvant chemotherapy prior to concurrent chemoradiotherapy for NPC. Sun et al. recently published a Phase III trial of neoadjuvant chemotherapy for locally advanced nasopharyngeal carcinoma. They report significantly improved failure-free survival at three years for the neoadjuvant chemotherapy arm [13].

Gross tumor volume downsizing for the radiation treatment is one of the intended goals of neoadjuvant chemotherapy. In this study, we did not quantify the effect of neoadjuvant chemotherapy on tumor shrinkage. However, the magnitude of dosimetric optimization after neoadjuvant chemotherapy is a topic of interest. A study of the volumetric effects of neoadjuvant chemotherapy is underway at our institution.

\section{Conclusions}

Locally advanced NPC patients have been treated with neoadjuvant chemotherapy at this center with favorable outcomes. Most patients could tolerate both neoadjuvant cisplatin-based chemotherapy and concurrent cisplatin chemotherapy. Higher doses of neoadjuvant chemotherapy and male sex were associated with receiving $>200 \mathrm{mg} / \mathrm{m}^{2}$ concurrent cisplatin. It appears that neoadjuvant cisplatin chemotherapy is tolerable prior to concurrent cisplatin with radiotherapy and increasing neoadjuvant cisplatin exposure is not a limiting factor for concurrent chemotherapy delivery. 


\section{Additional Information}

\section{Disclosures}

Human subjects: Consent was obtained by all participants in this study. BC Cancer Agency University of British Columbia issued approval H12-00751. Animal subjects: All authors have confirmed that this study did not involve animal subjects or tissue. Conflicts of interest: In compliance with the ICMJE uniform disclosure form, all authors declare the following:

Payment/services info: All authors have declared that no financial support was received from any organization for the submitted work. Financial relationships: All authors have declared that they have no financial relationships at present or within the previous three years with any organizations that might have an interest in the submitted work. Other relationships: All authors have declared that there are no other relationships or activities that could appear to have influenced the submitted work.

\section{References}

1. Baujat B, Audry H, Bourhis J, et al.: Chemotherapy in locally advanced nasopharyngeal carcinoma: an individual patient data meta-analysis of eight randomized trials and 1753 patients. Int J Radiat Oncol Biol Phys. 2006, 64:47-56. 10.1016/j.ijrobp.2005.06.037

2. Ma BB, Chan AT: Systemic treatment strategies and therapeutic monitoring for advanced nasopharyngeal carcinoma. Expert Rev Anticancer Ther. 2006, 6:383-394. 10.1586/14737140.6.3.383

3. Al-Sarraf M, LeBlanc M, Giri PG, et al.: Chemoradiotherapy versus radiotherapy in patients with advanced nasopharyngeal cancer: phase III randomized Intergroup study 0099. J Clin Oncol. 1998, 16:1310-1317. 10.1200/JCO.1998.16.4.1310

4. Chan AT, Leung SF, Ngan RK, et al.: Overall survival after concurrent cisplatin-radiotherapy compared with radiotherapy alone in locoregionally advanced nasopharyngeal carcinoma. J Natl Cancer Inst. 2005, 97:536-539. 10.1093/jnci/dji084

5. Chen L, Hu CS, Chen XZ, et al.: Concurrent chemoradiotherapy plus adjuvant chemotherapy versus concurrent chemoradiotherapy alone in patients with locoregionally advanced nasopharyngeal carcinoma: a phase 3 multicentre randomised controlled trial. Lancet Oncol. 2012, 13:163-171. 10.1016/S1470-2045(11)70320-5

6. Chen Y, Liu MZ, Liang SB, et al.: Preliminary results of a prospective randomized trial comparing concurrent chemoradiotherapy plus adjuvant chemotherapy with radiotherapy alone in patients with locoregionally advanced nasopharyngeal carcinoma in endemic regions of China. Int J Radiat Oncol Biol Phys. 2008, 71:1356-1364. 10.1016/j.ijrobp.2007.12.028

7. Kong L, Hu C, Niu X, Zhang Y, Guo Y, Tham IW, Lu JJ: Neoadjuvant chemotherapy followed by concurrent chemoradiation for locoregionally advanced nasopharyngeal carcinoma: interim results from 2 prospective phase 2 clinical trials. Cancer. 2013, 119:4111-4118. $10.1002 /$ cncr.28324

8. Hui EP, Ma BB, Leung SF, et al.: Randomized phase II trial of concurrent cisplatinradiotherapy with or without neoadjuvant docetaxel and cisplatin in advanced nasopharyngeal carcinoma. J Clin Oncol. 2009, 27:242-249.

9. Fountzilas G, Ciuleanu E, Bobos M, et al.: Induction chemotherapy followed by concomitant radiotherapy and weekly cisplatin versus the same concomitant chemoradiotherapy in patients with nasopharyngeal carcinoma: a randomized phase II study conducted by the Hellenic Cooperative Oncology Group (HeCOG) with biomarker evaluation. Ann Oncol. 2012, 23:427-435. 10.1093/annonc/mdr116

10. Yau TK, Lee AW, Wong DH, et al.: Induction chemotherapy with cisplatin and gemcitabine followed by accelerated radiotherapy and concurrent cisplatin in patients with stage IV(A-B) nasopharyngeal carcinoma. Head Neck. 2006, 28:880-887. 10.1002/hed.20421

11. Zhong YH, Dai J, Wang XY, Xie CH, Chen G, Zeng L, Zhou YF: Phase II trial of neoadjuvant docetaxel and cisplatin followed by intensity-modulated radiotherapy with concurrent cisplatin in locally advanced nasopharyngeal carcinoma. Cancer Chemother Pharmacol. 2013, 71:1577-1583. 10.1007/s00280-013-2157-2

12. Hamilton SN, Ho C, Laskin J, Zhai Y, Mak P, Wu J: Asian versus non-Asian outcomes in 
Cureus

nasopharyngeal carcinoma: a North American population-based analysis. Am J Clin Oncol. 2016, 39:575-580. 10.1097/COC.0000000000000091

13. Sun Y, Li WF, Chen NY, et al.: Induction chemotherapy plus concurrent chemoradiotherapy versus concurrent chemoradiotherapy alone in locoregionally advanced nasopharyngeal carcinoma: a phase 3, multicentre, randomised controlled trial. Lancet Oncol. 2016, 17:15091520. 10.1016/S1470-2045(16)30410-7 